\title{
STUDIES ON ANTIHYDROGEN ATOMS WITH THE ATRAP EXPERIMENT AT CERN*
}

\author{
M. Zieliński ${ }^{\mathrm{a}}$, D. GRZONKA ${ }^{\mathrm{b}}$, W. OelerT $^{\mathrm{b}, \mathrm{e}}$, T. SEFZiCK $^{\mathrm{b}}$

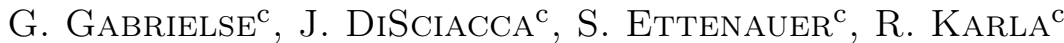 \\ K. MARABle ${ }^{c}$, M. MARshall ${ }^{c}$, E. TARdifF $^{c}$, E. Hessels $^{d}$ \\ C. Storry ${ }^{d}$, D. Fitzakerley ${ }^{d}$, M. George ${ }^{d}$, M. Weel $^{\mathrm{d}}$ \\ A. MÜLlERS ${ }^{\mathrm{e}}$, J. WALZ ${ }^{\mathrm{e}}$
}

for the ATRAP Collaboration

${ }^{a}$ The Marian Smoluchowski Institute of Physics, Jagiellonian University Reymonta 4, 30-059 Kraków, Poland

${ }^{\mathrm{b}}$ Institute für Kernphysik, Forschungszentrum Jülich, 52428 Jülich, Germany

${ }^{\mathrm{c}}$ Department of Physics, Harvard University, Cambridge MA 02138, USA

${ }^{\mathrm{d}}$ Department of Physics and Astronomy, York University Toronto M3J 1P3, Canada

e Institute für Physics, Johannes Gutenberg University Mainz 55099 Mainz, Germany

(Received August 26, 2013)

The CPT theorem predicts the same properties of matter and antimatter, however, in the nearby Universe, we observe a huge imbalance of matter and antimatter. Therefore, it is intriguing to measure the properties of particles and antiparticles in order to contribute to an explanation of this phenomena. In this article, we will describe the experimental efforts of the ATRAP Collaboration in order to test the CPT theorem using antihydrogen atoms.

DOI:10.5506/APhysPolBSupp.6.1093

PACS numbers: 37.10.De, 36.10.-k, 41.20.-q, 41.90. $+\mathrm{e}$

\section{Introduction}

In accordance with the Standard Model [1, 2] of particles and fields, there was a believe that the antimatter is an exact mirror of matter and all nature phenomena are invariant under the conjugation of charge $(\mathrm{C})$, parity $(\mathrm{P})$, time reversal $(\mathrm{T})$. This statement formally is enclosed in the CPT theorem [3] which is valid for local, Lorentz-invariant quantum field

* Presented at the Symposium on Applied Nuclear Physics and Innovative Technologies, Kraków, Poland, June 3-6, 2013. 
theories. Moreover, the CPT theorem implies the equal amount of matter and antimatter in the Universe. However, the experimental observations show a complete absence of antimatter (visible only as reaction products in cosmic rays) and dominance of matter in the observed Universe. The reason for this matter excess has not been discovered yet. Thus, particularly interesting are studies of particles and antiparticles properties in order to contribute in an explanation of this phenomena, and also experimentally test predictions of the CPT theorem.

One of the method for experimental test of the fundamental CPT symmetry was proposed by Gabrielse back in 1987 [4]:

"For me, the most attractive way ... would be to capture the antihydrogen in a neutral particle trap such as has been used for neutrons and neutral atoms. The objective would be to then study the properties of a small number of [antihydrogen] atoms confined in the neutral trap for a long time."

Particularly, this could be accomplished by investigating the possible spectroscopic differences between the antihydrogen $(\overline{\mathrm{H}})$ and the hydrogen atoms $(\mathrm{H})$. Such spectroscopy could compare $\overline{\mathrm{H}}$ and $\mathrm{H}$ at a higher precision than the most stringent CPT test with leptons [5] and baryons [6], and cold trapped antihydrogen atoms would also allow for precise measurement of the gravitational interaction between matter and antimatter [7, 8]. However, a prerequisite for such precise studies is confining a suitable number of ground state $\overline{\mathrm{H}}$ atoms.

Eight years after the initial proposals were made the first nine antihydrogen atoms in the world were produced by a group leaded by W. Oelert at the LEAR/CERN facility [9]. In this experiment the production of $\overline{\mathrm{H}}$ atoms was predominantly mediated by the $e^{+} e^{-}$pair creation in an antiproton-nucleus interaction. However, all identified antimatter atoms were produced with relativistic velocities making them much too hot to be investigated.

Nowadays the experiments ALPHA [10], ASACUSA [11] and ATRAP at the AD decelerator at CERN are producing antihydrogen atoms routinely. The ATRAP Collaboration has produced antihydrogen atoms by two different techniques: two body recombination [12] and laser controlled charge exchange [13], also within a combined Penning-Ioffe trap [14], and more recently had demonstrated confinement of $\overline{\mathrm{H}}$ in a Ioffe trap for long enough to ensure the atoms will reach their ground state [15] and be suitable for spectroscopic measurements.

\section{ATRAP apparatus and antihydrogen production}

The ATRAP apparatus is a system of many different components used to produce, trap and detect antihydrogen atoms. The main part of the setup 
is a $1 \mathrm{~T}$ superconducting solenoid in which a Penning trap is placed, with a Ioffe trap superimposed upon it. The system of traps is surrounded by a tube of scintillating fiber detectors with 4 layers and outside the solenoid 2 layers of scintillating paddle detectors are installed which together are used for annihilation detection. In an event of proton-antiproton annihilation approximately three charged pions are produced [16, 17], each with energies of few hundred $\mathrm{MeV}$. The minimum ionizing pions leave the Penning trap giving signals in the scintillating fiber and the outer paddle detector. It is also possible to register electron-positron annihilation which results in a two $511 \mathrm{keV}$ gamma quanta, however with the much lower efficiency than for charged particles. For monitoring, steering and focusing of the primary $\bar{p}$ beam silicon detectors and Parallel Plate Avalanche Counters are utilized [18, 19]. Moreover, the ATRAP apparatus is equipped with non-destructive detection of particle clouds with the radio-frequency techniques [20, 21].

Separately, a positron production system is used from which $e^{+}$are transferred to the experimental region by a $9 \mathrm{~m}$ long magnetic transfer line. A schematic view of the ATRAP apparatus is shown in Figs. 1 and 2.

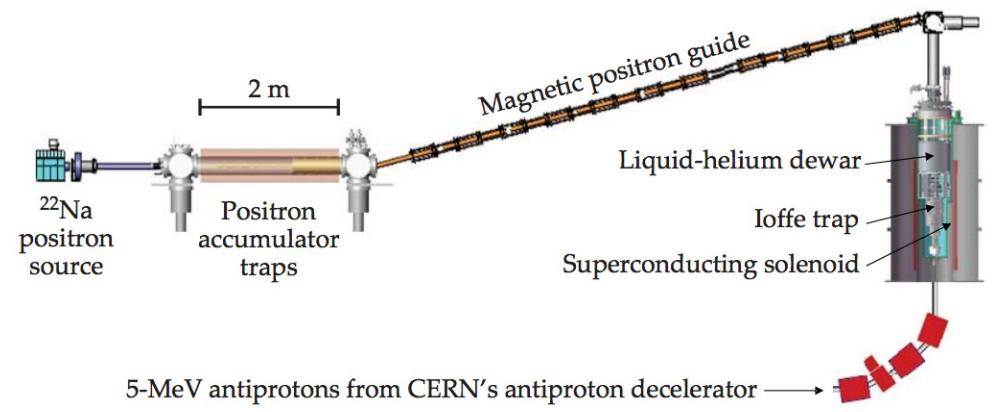

Fig. 1. Overview of the ATRAP apparatus operating at the Antiproton Decelerator (AD) at CERN.

To produce antihydrogen atoms with the two-body recombination method, one needs to store antiprotons and positrons. For the ATRAP experiment, every $90 \mathrm{~s}$, approximately $3 \times 10^{7}$ antiprotons with energies of $5.3 \mathrm{MeV}$ are delivered by the Antiproton Decelerator (AD). Those are still too fast for trapping and, therefore, they are slowed down in a beryllium degrader and trapped in a Penning trap with an efficiency below a per cent, where they are further cooled by collisions with electrons. In parallel, positrons coming from the $\beta^{+}$decay of ${ }^{22} \mathrm{Na}$ source are accumulated in a separate trap, and on demand transferred in a pulse of $10^{7} e^{+}$into a cryogenic nested Penning trap where they can mix with antiprotons. As the positrons emit synchrotron radiation, they reach the thermal equilibrium with the trap in several minutes in the $1 \mathrm{~T}$ magnetic field. The antiprotons cool further in collisions with 
positrons and can collide with two positrons to form antihydrogen atoms, where the second positron plays a role of spectator to meet the energy and the momentum conservation law. In order to confine the produced antihydrogen atoms, a Ioffe trap generates strong magnetic field gradients, which holds neutral $\overline{\mathrm{H}}$ if their energy is low enough.

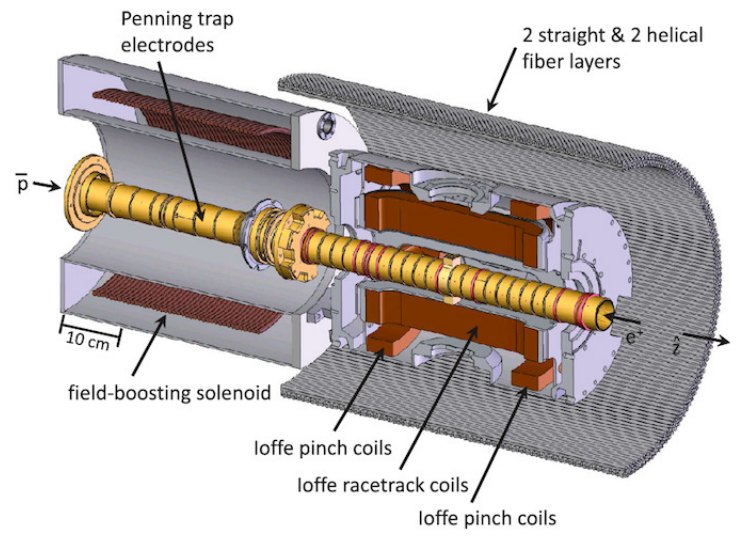

Fig. 2. Schematic view of the ATRAP electrodes of the Penning trap (to store $\bar{p}$ and $e^{+}$) and Ioffe trap (to store $\overline{\mathrm{H}}$ ). The solenoid is not shown in this picture but it gives $1 \mathrm{~T}$ magnetic field along the trap vertical $z$ axis.

The production of low energy, cold antihydrogen atoms can be accomplished also by the laser controlled charge exchange method which was demonstrated at ATRAP and it is a very promising method. In this approach, caesium atoms (Cs) are excited by a green laser into a Rydberg states. Next, $\mathrm{Cs}^{*}$ atoms collide with positrons to form excited positronium atoms, which then collide with trapped antiprotons to form antihydrogen atoms.

\section{Recent results}

The ATRAP Collaboration as well as the other AD experiments made big steps during the past years, getting the dream about the spectroscopy of antihydrogen atoms closer to reality. One of the difficulties in producing cold antihydrogen is the ability of careful manipulation of positron and antiproton clouds to avoid any heating of the system. One of the method for acquiring such cool antiprotons is to utilize the electron cooling method [22]. The ATRAP Collaboration has developed a method of centrifugal separation of antiprotons and electrons during the $e^{-}$cooling process [23]. Observed separation is large even for large number of electrons $\left(N \approx 10^{8}\right)$, with high densities $\left(n \approx 10^{8} / \mathrm{cm}^{3}\right)$, and modest magnetic fields, and finally with up to $10^{7} \bar{p}$. The separation was easily visible with the method which permits to 
gently remove well controlled fraction of $e^{-}$from the trap. Figure 3 (top) shows that $e^{-}$escape the trap before the $\bar{p}$ as the applied trapping voltage is ramped down. Figure 3 (bottom) shows that the much larger fraction of $\bar{p}$ remains in the trap.
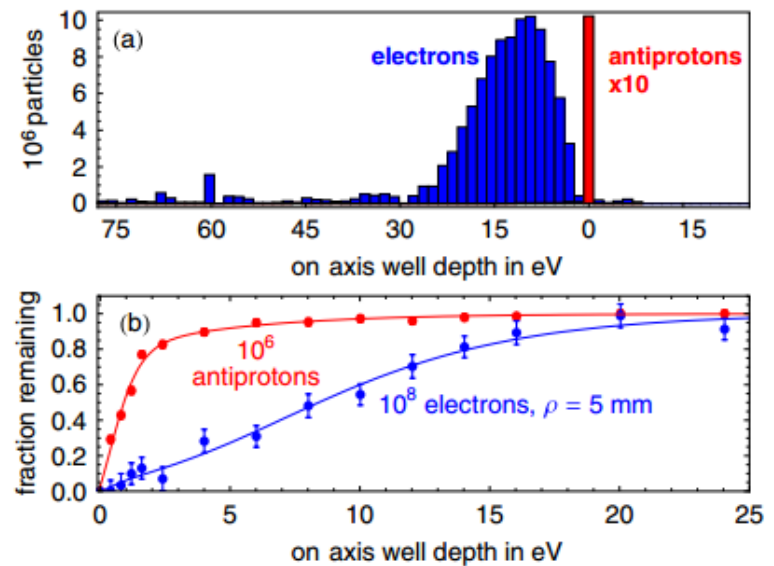

Fig. 3. (Top) Number of antiprotons and electrons escaping the trap each time the applied potential is reduced non-adiabatically in $2 \mathrm{~V}$ steps. (Bottom) Fraction of antiprotons and electrons remaining after the well depth is ramped adiabatically to the indicated value.

Another important step was the demonstration of the adiabatic cooling of the antiprotons as a very effective way of cooling particles to very low temperatures [24]. We have shown the ability of cooling up to $10^{6}$ antiprotons to temperature of $3.5 \mathrm{~K}$. Moreover, no $\bar{p}$ are lost during that process which is a very important advantage for such rare particles.

ATRAP recently demonstrated a long-time confinement of antihydrogen atoms formed via three-body recombination method. In order to work out the most efficient way of producing cold $\bar{H}$, trials with different driving forces applied to make $\bar{p}$ pass through the $e^{+}$were done. Production and trapping of antihydrogen lasted from $2 \mathrm{~ms}$ to $15 \mathrm{~min}$. The produced $\overline{\mathrm{H}}$ atoms stayed confined as long as its radiative decay took them to another low field seeking state whose kinetic energy is less than the Ioffe trap well depth for the state. The rest of the antiprotons and positrons which did not form atoms are cleared out by an axial electric field. The antihydrogen atoms remaining in the trap are released by quenching the superconducting racetrack coils of the Ioffe trap. The $\overline{\mathrm{H}}$ released from the Ioffe trap annihilated producing pions which are then registered by scintillating detector system. The efficiency of $\bar{p}$ annihilation detection in the coincidence between paddles and fiber is $33 \%$, with the cosmic ray background of $1.7 \mathrm{~Hz}$. Figure 4 (top) shows the sum 
of the detector counts for the 20 trials in $1 \mathrm{~s}$ intervals. The peak indicates trapping of $105 \pm 21$ antihydrogen atoms in the $375 \mathrm{mK}$ quadrupole Ioffe trap, on the level of 6 standard deviations above the expected background. This corresponds to the $5 \pm 1$ simultaneously trapped $\overline{\mathrm{H}}$ per trail, stored in the trap between 15 to $1000 \mathrm{~s}$. Additionally, we have performed 20 controlled trails without $\overline{\mathrm{H}}$ production and trapping. Figure 4 (bottom) shows that the sudden flux change from quenching the trap does not induce a false coincidence signal.
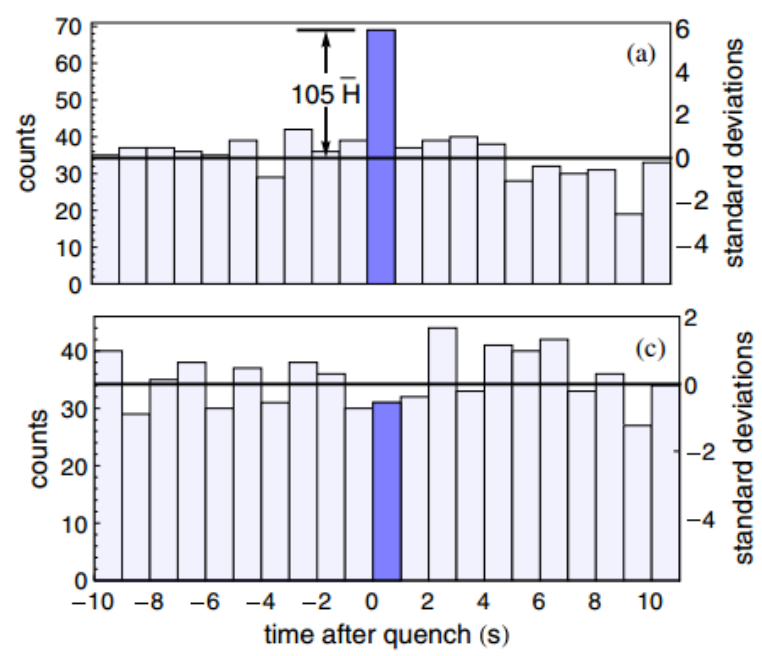

Fig. 4. (Top) Detector counts in $1 \mathrm{~s}$ time intervals for 20 trials in which the Ioffe trap is turned off between $t=0$ and $t=1$ to release trapped $\overline{\mathrm{H}}$. (Bottom) 20 controled trials with no trapped $\overline{\mathrm{H}}$. The solid line on both plots shows average number of counts from cosmic rays.

\section{Conclusions}

The presented results of the ATRAP Collaboration show clearly the progress towards the final goal of spectroscopic studies on antihydrogen atoms, and encourage for further work.

The centrifugal separation of simultaneously trapped $\bar{p}$ and $e^{-}$is clearly observed and gives a new approach for isolating the low energy antiprotons for producing mixtures of $\bar{p}$ and $e^{-}$. Furthermore, centrifugal separation introduces a new method to remove $e^{-}$with less heating and with good control of ratio of $\bar{p}$ and $e^{-}$. Moreover, we demonstrated the possibility of adiabatic cooling of antiprotons to $T=3.5 \pm 0.7$ the lowest directly measured $\bar{p}$ temperature. The most important result is the simultaneously trapping of antihydrogen atoms where we observed $105 \overline{\mathrm{H}}$ in the $375 \mathrm{mK}$ quadruple Ioffe trap. 
We are also very optimistic as to increasing the number of produced antihydrogen atoms due to use of a new Ioffe trap with quadruple/octuple field configurations and fast ramping ability for the future experiments. Furthermore, an extension of the AD facility will be done by an additional decelerator ELENA [23] which will increase the trapping efficiency by orders of magnitude resulting in more rapid progress of the experiments.

This work was supported by the NSF and AFOSR of the US, the BMBF, DFG, and DAAD of Germany, and the NSERC, CRC and CFI of Canada, and the Polish National Science Centre under the grant number 2011/03/N/ ST2/02653.

\section{REFERENCES}

[1] S.L. Glashow, Nucl. Phys. 22, 579 (1961).

[2] S. Weinberg, Phys. Rev. Lett. 19, 1264 (1967).

[3] G. Lüders, Ann. Phys. 2, 1 (1957).

[4] G. Gabrielse, in: Fundamental Symmetries, edited by P. Bloch, P. Pavlopoulos, R. Klapisch, Plenum, New York 1987, pp. 59-75.

[5] R.S. Van Duck et al., Phys. Rev. Lett. 59, 26 (1987).

[6] G. Gabrielse et al., Phys. Rev. Lett. 82, 3198 (1999).

[7] G. Gabrielse, Hyperfine Interact. 44, 349 (1988).

[8] J. Walz, T. Hänsch, Gen. Relativ. Gravitation 36, 561 (2004).

[9] G. Baur et al., Phys. Lett. B368, 251 (1996).

[10] W. Beitsche et al., Nucl. Instrum. Methods Phys. Res. A 566, 746 (2006).

[11] M. Hori, Nucl. Phys. A692, 119C (2001).

[12] G. Gabrielse et al., Phys. Rev. Lett. 89, 213401 (2002).

[13] C. Storry et al., Phys. Rev. Lett. 93, 263401 (2004).

[14] G. Gabrielse et al., Phys. Rev. Lett. 100, 113001 (2008).

[15] G. Gabrielse et al., Phys. Rev. Lett. 108, 113002 (2012).

[16] C. Amsler, Rev. Mod. Phys. 70, 1293 (1998).

[17] C. Amsler, F. Myher, Annu. Rev. Nucl. Part. Sci. 41, 219 (1991).

[18] H. Stelzer, Nucl. Instrum. Methods 133, 409 (1976).

[19] G. Gabrielse et al., Phys. Rev. A40, 481 (1989).

[20] D.J. Wineland, H.G. Dehmelt, J. Appl. Phys. 46, 919 (1975).

[21] L.S. Brown, G. Gabrielse, Rev. Mod. Phys. 58, 233 (1986).

[22] G. Gabrielse et al., Phys. Lett. B548, 140 (2002).

[23] G. Gabrielse et al., Phys. Rev. Lett. 105, 213002 (2010).

[24] G. Gabrielse et al., Phys. Rev. Lett. 106, 073002 (2011).

[25] [AD User Community] J. Alsner et al., "ELENA: An Upgrade to the Antiproton Decelerator", CERN-SPSC-2009-026; SPSC-P-338, 2010. 